\title{
Recognizing blood pressure patterns in sedated critically ill patients on mechanical ventilation by spectral clustering
}

\author{
Shengjun Liu ${ }^{1 \#}$, Longxiang Su ${ }^{1 \#}$, Xin Liu ${ }^{2}$, Xueqian Zhang ${ }^{2}$, Zuyu Chen ${ }^{2}$, Chun Liu ${ }^{3}$ Na Hong ${ }^{3}$, Yali Li $^{2}$, \\ Yun Long ${ }^{1}$
}

${ }^{1}$ Department of Critical Care Medicine, State Key Laboratory of Complex Severe and Rare Diseases, Peking Union Medical College Hospital, Chinese Academy of Medical Science \& Peking Union Medical College, Beijing, China; ${ }^{2}$ Department of Electronic Engineering, Tsinghua University, Beijing, China; ${ }^{3}$ Digital Health China Technologies Co., Ltd., Beijing, China

Contributions: (I) Conception and design: S Liu, L Su; (II) Administrative support: Y Long, Y Li; (III) Provision of study materials or patients: S Liu, L Su; (IV) Collection and assembly of data: C Liu, N Hong, S Liu; (V) Data analysis and interpretation: X Liu, X Zhang, Z Chen; (VI) Manuscript writing: All authors; (VII) Final approval of manuscript: All authors.

\#These authors contributed equally to this work.

Correspondence to: Dr. Yun Long. Department of Critical Care Medicine, Chinese Academy of Medical Science \& Peking Union Medical College,

Beijing, China. Email: ly_icu@aliyun.com; Dr. Yali Li. Department of Electronic Engineering, Tsinghua University, Beijing, China.

Email: liyali13@tsinghua.edu.cn.

Background: Blood pressure is a critical therapeutic goal in intensive care unit (ICU). One important factor influencing blood pressure are analgesia and sedation. Analgesic and sedative drugs are commonly used in critically ill patients. These drugs affect blood pressure by reducing the tension of the venous system, the cardiac preload, and cardiac output and inhibiting cardiac functions. Consequently, vasoactive agents are commonly used to increase blood pressure. The indications for the usage of vasoactive agents are unequivocal. However, opinions on when to stop raising blood pressure vary. This study explored the relationship between blood pressure and sedation.

Methods: Patients in the Multiparameter Intelligent Monitoring in Intensive Care-III (MIMIC) database who had received mechanical ventilation, had been administered sedative analgesics during their ICU stay, and met the inclusion criteria were included in this study. The mean arterial pressure (MAP) tendency patterns were identified using spectral clustering and visualized using the t-distributed Stochastic Neighbor Embedding (t-SNE) algorithm. The 28-day mortality rates of patients with different MAP patterns during their first 24 hours in the ICU and their sedation levels were calculated in the crosstab.

Results: Fourteen thousand seven hundred and eighty-five patients from the MIMIC-III database were included in this study. Three MAP patterns were identified by spectral clustering. The median MAP of the low, moderate, and high MAP groups was 71.2, 80.4, and $97.6 \mathrm{mmHg}$, respectively. The 28-day mortality rate of patients in the moderate MAP group (13.0\%) was lower than that of patients in the low (16.6\%) and high (15.6\%) MAP groups. No difference was found in the 28-day mortality rate between the low and high MAP groups. Dynamic changes in blood pressure at different sedation depths were also examined. Notably, compared with light and moderate sedation level, patients in the deep sedation group, especially those in the high MAP group (48.5\%), had a higher 28-day mortality rate (36.5\%).

Conclusions: Low MAP in the first 24 hours in ICU indicates a high possibility of poor prognosis for critically ill patients on mechanical ventilation. For patients under deep sedation, maintaining a high mean arterial pressure also indicates poor prognosis. A personalized MAP target should be determined according to the severity of illness and level of sedation for each patient.

Keywords: Blood pressure patterns; spectral clustering; t-distributed stochastic neighbor embedding (t-SNE); mechanical ventilation; sedation administration 
Submitted May 29, 2021. Accepted for publication Aug 06, 2021.

doi: 10.21037/atm-21-2806

View this article at: https://dx.doi.org/10.21037/atm-21-2806

\section{Introduction}

In the intensive care unit (ICU), patients suffer from discomfort and pain, which are mainly attributable to hypoxia, shock, high fever, visceral pain, and surgical incisions. Additionally, studies have shown that various treatment procedures and noisy medical environments can further deteriorate the surroundings of patients $(1,2)$. Such factors may increase patients' anxiety, irritability, pain and may even make them delirious. In addition to negative subjective emotions, these factors may also lead to changes in patients' physiological states, increase the burden placed on organ functions, and worsen patients' condition in severe cases (3). Thus, sedatives are frequently administered to critically ill patients in the ICU to relieve anxiety, reduce the stress of being mechanically ventilated, and prevent agitation-related harm $(4,5)$.

Due to their pharmacological features, the use of sedatives may increase morbidity (6). The depth of sedation is associated with delirium, increase the duration of mechanical ventilation, increase the length of stay, and decrease survival (7). Accumulating evidences have shown the hemodynamic effects of sedation (8). As blood pressure changes, analgesia and sedation drugs may reduce the tension of the venous system, reduce the tension volume, and change patient's volume state, thereby reducing the cardiac preload and cardiac output and lowering blood pressure (9). Additionally, Analgesic and sedative drugs can inhibit cardiac functions due to their negative chronotropic and negative inotropic effects $(10,11)$.

Consequently, doctors may use vasoactive agents to increase blood pressure in the ICU. However, there is debate on whether maintaining a high mean arterial pressure (MAP) is essential for sedated patients in intensive care unit (12). Guidelines provide indications on the use of vasoactive agents (13) but fail to indicate when blood pressure should stop being elevated.

This study used the spectral clustering method to examine MAP patterns in sedated ICU patients and realized dimensionality reduction by $\mathrm{t}$-distributed stochastic neighbor embedding ( $t-\mathrm{SNE}$ ) algorithm. Clinical features were extracted to identify any standard or feature that determines the patients' optimum blood pressure and sedation depth.

We present the following article in accordance with the STROBE reporting checklist (available at https://dx.doi. org/10.21037/atm-21-2806).

\section{Methods}

\section{Data set}

The Medical Information Mart for Intensive Care-III (MIMIC-III) database is a free database comprising deidentified health-related data. The database comprises the data of over 40,000 patients who stayed in critical care units at the Beth Israel Deaconess Medical Center between 2001 and 2012 (14). We used the data of 14,785 adults from the MIMIC-III database who had received mechanical ventilation and had been administered sedative analgesics during their ICU stay. Patients' data were extracted from the MIMIC-III database for the study if they met the following inclusion criteria: (I) The patient was aged over 18 years, had complete medical records, and had received medical ventilation; and/or (II) the patient had received any of the following 5 sedative or analgesic drugs: fentanyl, midazolam, morphine, propofol, and dexmedetomidine. The patient selection process is shown in Figure 1. For missing values in the MAP data, we applied the Akima method (15), as it provides a natural and smooth interpolation that builds a continuously differentiable subspline from piece-wise cubic polynomials.

\section{Analysis of analgesia and sedation depth}

The Richmond-Agitation-Sedation Scale (ASS) (16) and the Riker Sedation-Agitation Scale (17) reflect the degree of sedation of a patient. The lower the score, the better the sedation effect. In the MIMIC-III database, sedation depth is evaluated according to these 2 scales. Based on previous clinical studies and experiences, we divided patients into the 3 categories to reflect different degrees of sedation (deep sedation, moderate sedation, and light sedation) based on Richmond-ASS scores of $[-5,-3],[-3,0]$, and $[0,+4]$, and Riker Sedation-Agitation Scale scores of 1 and 2, 3 and 4, and $\geq 5$ respectively (18). 


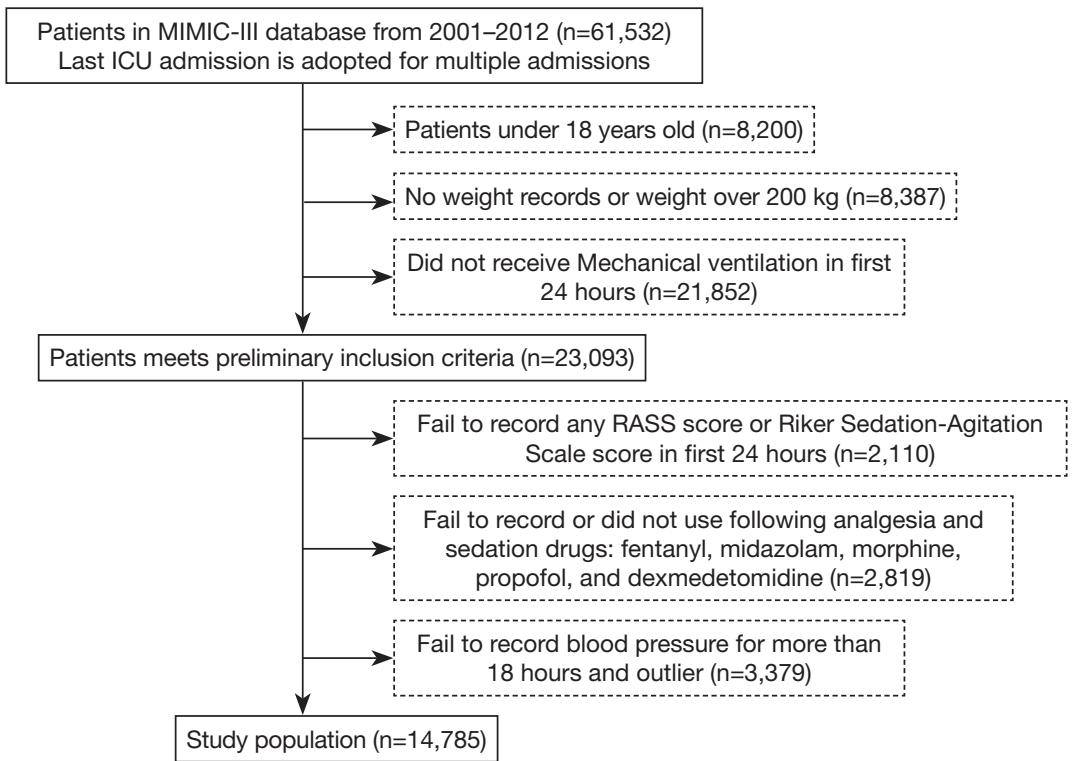

Figure 1 Flow chart of patient selection. MIMIC-III, Medical Information Mart for Intensive Care; ICU, intensive care unit; RASS, Richmond agitation-sedation scale.

\section{Clustering analysis}

We applied spectral clustering (19) method to explore the patterns of the MAP data. As a flexible and computationally efficient approach, spectral clustering does not make any assumption on the form of the data clusters; thus, it was an appropriate method for clustering the MAP data. The method uses the spectrum of the affinity matrix, which is constructed by computing a graph of the nearest neighbors, to classify MAP data points into 3 groups according to their similarities. From the graph cut point of view, each data point can be regarded as a graph's vertex in a high-dimensional space. Spectral clustering aims to find a partition in the graph for which the edges within the same groups have a high weight while the edges between different groups have a low weight. The algorithm can be formulated as:

Minimize Graphcut

$$
\left(A_{1}, \ldots, A_{k}\right)=\frac{1}{2} \sum_{i=1}^{k} \sum_{m \in A_{i}, n \in \bar{A}_{i}} w_{m n}
$$

where $A_{1}, \ldots, A_{k}$ denote the partition of the graph, $\bar{A}_{i}$ complement, and represent the weight between data point $\mathrm{m}$ and data point $\mathrm{n}$.

As the ground-truth labels were not known, we used a silhouette coefficient to evaluate the clustering quality. This coefficient measures how similar a data point is to its cluster compared to other clusters (20). the silhouette coefficient is given as:

$$
s=\frac{d_{b}-d_{a}}{\max \left(d_{a}, d_{b}\right)}
$$

where $d_{a}$ denotes the mean distance between a data point and all other points in the same cluster, and $d_{b}$ is the mean distance between a data point and all other points in the next nearest cluster. In our study, the silhouette coefficient was 0.21 , which indicates that the clusters were relatively dense and well separated.

\section{t-distributed stochastic neighbor embedding}

t-SNE is a non-linear dimensionality reduction algorithm that approximates a simple form of spectral clustering and is suitable for the dimensionality reduction of highdimensional data into 2 or 3 dimensions for visualization. $\mathrm{t}$-SNE converts the similarity between data points into probabilities and models the distribution of each data point's neighbors (the term 'neighbors' refers to a set of data points close to each other). The similarity in the original high-dimensional space is represented by the Gaussian distribution, and the similarity in the embedding space is represented by the t-distribution. The ultimate goal is to minimize the gap between these 2 distributions for all points. The Kullback-Leibler (KL) divergence of the joint 
Table 1 Basic patient characteristics of the included patient population

\begin{tabular}{lc}
\hline Parameters & Values \\
\hline Age (years) & $63.8 \pm 15.9$ \\
Male gender & $9,120(61.7)$ \\
SAPS II score & $37[29-47]$ \\
SOFA score & $5[3-7]$ \\
Mortality (28 days) & $14.8 \%$ \\
Sedation depth & \\
Deep & $1,610(10.9)$ \\
Moderate & $12,742(86.2)$ \\
Light & $433(2.9)$ \\
Sedatives/analgesics usage & \\
Dexmedetomidine & $834(5.6)$ \\
Fentanyl & $5,828(39.4)$ \\
Midazolam & $4,010(27.1)$ \\
Morphine & $3,936(26.6)$ \\
Propofol & $12,691(85.8)$ \\
\hline SAPS II, Simplied Acute Physiolog & \\
\hline
\end{tabular}

SAPS II, Simplified Acute Physiology Score II; SOFA, Sequential Organ Failure Assessment.

probability of the original space and the embedding space is used to evaluate the quality of the visualization; that is, the function of the $\mathrm{KL}$ divergence is used as the loss function, which is given as:

$$
K L(P \| Q)=\sum_{i} \sum_{j} P_{i j} \log \frac{P_{i j}}{Q_{i j}}
$$

where $P$ and $Q$ are the distribution function of data in the original space and embedding space. The loss function is then minimized by the gradient descent method, and finally, the convergence results can be obtained. The distribution of MAP data are shown in a 2-dimensional space.

\section{Statistical methods}

Statistical analyses were performed using SPSS Statistics under the Windows system (version 19.0, IBM Corporation, Armonk, NY). The clustering analysis and t-SNE were implemented based on Python programming and Scikitlearn library. The student's $t$-test or the Wilcoxon ranksum test was used to compare the continuous variables. The categorical data were compared using the chi-square test or Fisher's exact test. A one-way analysis of variance among groups was used to compare differences in continuous parametric variables with normal distributions, and the Kruskal-Wallis test was used to compare differences in continuous parametric variables with abnormal distributions. Differences in the variables between the groups were considered statistically significant at the level of $\mathrm{P}<0.001$.

\section{Ethical statement}

This study is carried out according to the provisions of the Declaration of Helsinki (revised in 2013). Data in MIMICIII database was deidentified in accordance with Health Insurance Portability and Accountability Act (HIPAA) standards using structured data cleansing and date shifting. Protected health information was removed from free text fields. The components of this deidentification system are continually expanded as new data is acquired.

\section{Results}

\section{Patient characteristics}

14,785 patients from the MIMIC-III database were included in this study. The cohort comprised 5,665 women (38.3\%) and 9,120 men $(61.7 \%)$ with a mean age of $63.8 \pm 15.9$ years. The simplified acute physiology score (SAPS II) score and sequential organ failure assessment (SOFA) score are 37 [interquartile range (IQR): 29 to 47] and 5 (IQR: 3 to 7), respectively. The overall 28 -day mortality of the included patients was $14.8 \%$. According to the analysis of sedation depth, the number of patients in deep sedation, moderate sedation, light sedation was $1,610,12,472$, and 433 , respectively (Table 1).

\section{Clustering of blood pressure patterns}

Figure 2 shows the visualization of the clustering results, which was produced by reducing the dimensionality of highdimensional MAP data to 2 dimensions using the t-SNE algorithm. The blood pressure vectors of the 14,785 patients were classified into 3 categories.

\section{Characteristics exploration of the 3 blood pressure patterns}

According to the clustering, Figure 3 shows the MAP tendency of overall patients and the 3 MAP patterns for 
patients in the initial 6-24 hours in the ICU. The mean MAPs and their $95 \%$ confidence intervals are depicted by the full lines and shadow areas. The trend is similar between the deep, moderate, and light sedation groups. The clinical characteristics of the 3 groups are shown in Table 2 . Notably, the median, mean MAP of the low, moderate, and high MAP group was $71.2,80.4$, and $97.6 \mathrm{mmHg}$, respectively. The 28-day mortality rate of patients in the moderate MAP group (13.0\%) was statistically lower than that of patients in the low MAP (16.6\%) and high MAP (15.6\%) groups. However, there was no statistically significant difference in the 28-day mortality rate between the low and high MAP groups.

The 28-day mortality rates of patients are set out in Table 3. Patients in the deep sedation group, especially those in the high MAP group, had higher 28-day mortality compared with other groups. Additionally, the mortality of

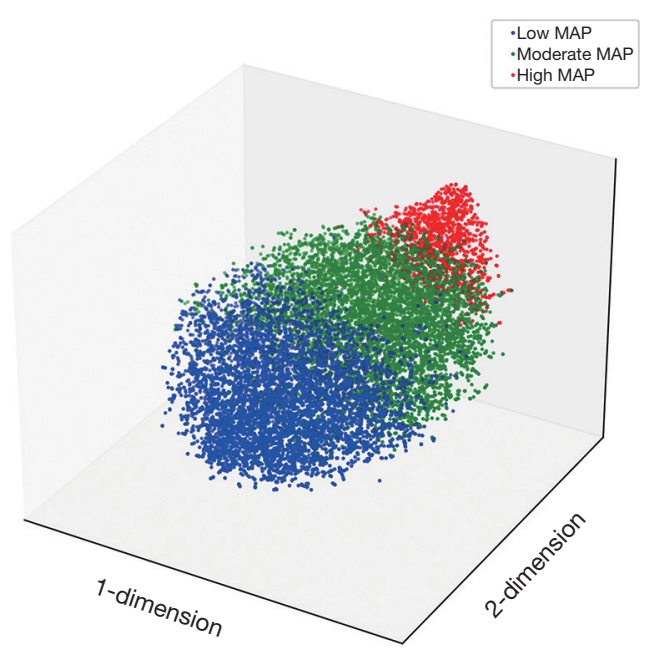

Figure 2 t-SNE visualization analysis of clustering results for blood pressure patterns. t-SNE, t-distributed stochastic neighbor embedding; MAP, mean arterial pressure. patients in the moderate sedation and moderate MAP group was $10.2 \%(622 / 6,128)$, which was statistically significantly lower than the mortality of patients overall $(2,181 / 14,785)$ $(\mathrm{P}<0.001)$.

\section{Discussion}

Using the data of the sedated mechanical ventilation patients, a 3-way classification of the MAP tendency pattern was made by spectral clustering and visualized by the t-SNE algorithm. We found that the 28-day mortality rate of patients in the moderate pattern MAP group was lower than that of patients in the low and high pattern MAP groups. However, there was no difference in the 28-day mortality rate between the low and high MAP groups. Additionally, low MAP was associated with the severity of illness (i.e., higher SAPS II and SOFA scores), a deep sedation level, and more usage of sedative/analgesic medications. Thus, clinicians must balance the depth of analgesia and sedation against the adverse hemodynamic effects of the drugs.

Unsurprisingly, compared to the same sedation level, patients with low MAP had a higher rate of 28-day mortality compared with other groups. The harms of hypotension in critically ill patients are obvious. In a retrospective analysis of 110 United States hospitals, researchers found that for every 1 unit increase in a timeweighted average MAP $<65 \mathrm{mmHg}$, the odds of in-hospital mortality increased by $11.4 \%(\mathrm{P}<0.001)$, the odds of acute kidney injury increased by $7.0 \%(\mathrm{P}<0.001)$, and the odds of myocardial injury increased by $4.5 \%(\mathrm{P}=0.03)(21)$. However, in our study, we found that even if the SAPS II and SOFA score was statistically higher in the low MAP group, the 28-day mortality rate of patients with high MAP was not statistically lower than that of patients with low MAP ( $\mathrm{P}=0.360)$. Thus, patients do not appear to benefit from aimless blood pressure elevating.
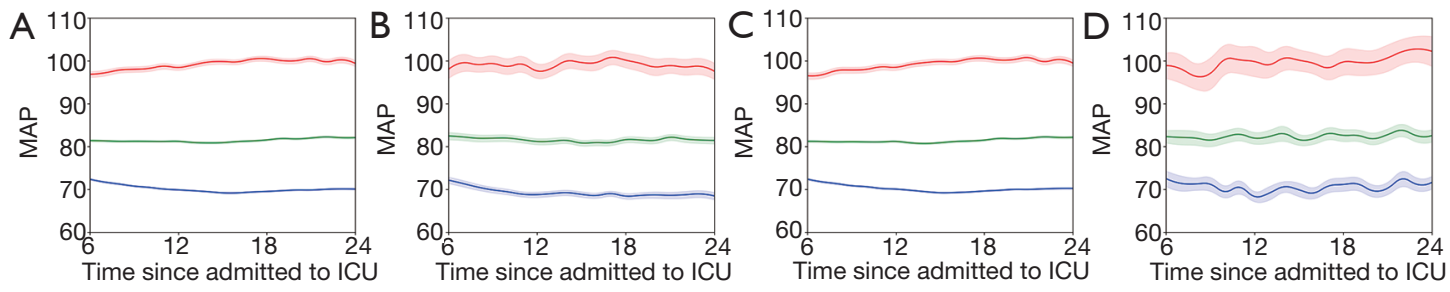

Figure 3 Visualization of MAP of (A) overall, (B) deep sedation, (C) moderate sedation, (D) light sedation patients in initial 6-24 hours in ICU. The red, green and blue line represents high, moderate and low MAP groups respectively; unit of $\mathrm{x}$ axis is hour. MAP, mean arterial pressure; ICU, intensive care unit. 
Table 2 Description of clinical characteristics of three blood pressure patterns

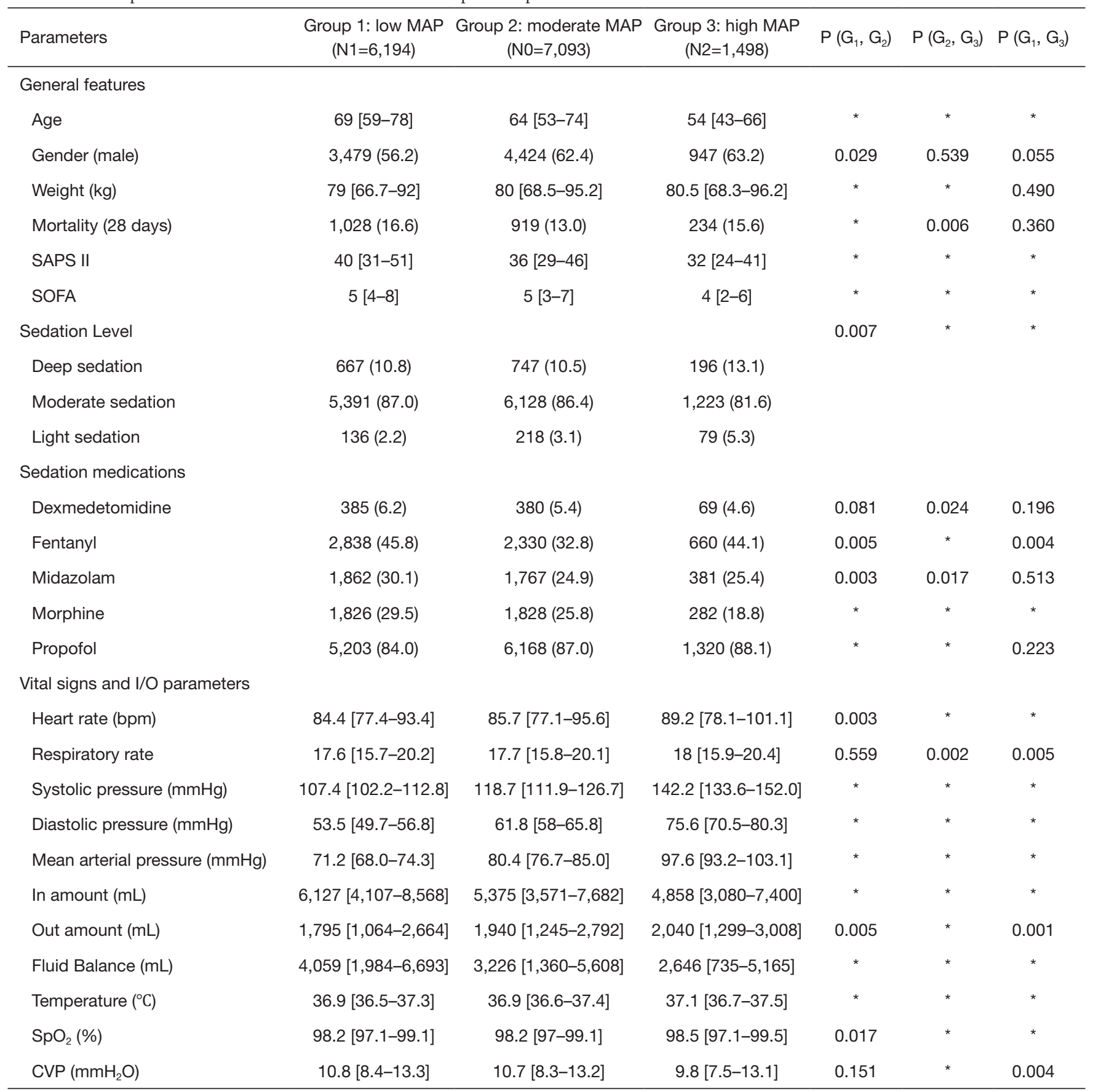

Table 2 (continued) 
Table 2 (continued)

\begin{tabular}{|c|c|c|c|c|c|c|}
\hline Parameters & $\begin{array}{l}\text { Group 1: low MAP } \\
\qquad(\mathrm{N} 1=6,194)\end{array}$ & $\begin{array}{c}\text { Group 2: moderate MAP } \\
(\mathrm{N} 0=7,093)\end{array}$ & $\begin{array}{l}\text { Group 3: high MAP } \\
\qquad(\mathrm{N} 2=1,498)\end{array}$ & $P\left(G_{1}, G_{2}\right)$ & $P\left(G_{2}, G_{3}\right)$ & $P\left(G_{1}, G_{3}\right)$ \\
\hline \multicolumn{7}{|l|}{ Laboratory examinations } \\
\hline White blood cell $\left(\times 10^{9}\right)$ & $12.2[9.3-16]$ & $11.7[8.9-15.1]$ & $11.4[8.4-14.9]$ & * & * & 0.008 \\
\hline Hematocrit (\%) & 29.9 [27.5-32.9] & 31.4 [28.4-35.1] & 33.9 [30.1-38.4] & * & * & * \\
\hline Platelet $\left(\times 10^{9}\right)$ & $179[137-241]$ & $188.5[141-251]$ & 211 [144-276] & * & * & * \\
\hline CRP (mg/L) & $77.4[13.4-150.7]$ & $41.1[7.4-123.3]$ & $18.9[7.7-56.4]$ & 0.230 & 0.009 & 0.079 \\
\hline ALT (U/L) & 28 [17-61] & 30 [18-71] & 35 [19-120.5] & 0.003 & * & * \\
\hline AST (U/L) & 47 [27-105] & 44 [26-104] & 48 [25-158] & 0.303 & 0.199 & 0.035 \\
\hline BUN (mg/dL) & 18.5 [13.5-29.5] & 17 [12.5-25.3] & 16.5 [11.5-25] & * & * & 0.004 \\
\hline Lactate (mmol/L) & $2.0[1.4-2.8]$ & $1.9[1.4-2.7]$ & $2.0[1.4-3.0]$ & * & 0.898 & 0.060 \\
\hline Potassium (mmol/L) & $4.3[4-4.6]$ & $4.2[3.9-4.5]$ & $4.0[3.7-4.3]$ & * & * & * \\
\hline Sodium (mmol/L) & $138[136-140]$ & 138 [136-140] & 139 [137-142] & * & * & * \\
\hline Chloride (mmol/L) & 107 [104-110] & 106.3 [103-109] & 105.2 [102-108] & * & * & * \\
\hline $\mathrm{HCO}_{3}^{-}(\mathrm{mmol} / \mathrm{L})$ & 23.5 [21-25.3] & 23.7 [21.5-25.7] & 24 [21.5-26.3] & * & * & 0.022 \\
\hline Base excess (mmol/L) & $2.3[1.5-3.3]$ & $2.3[1.5-3.5]$ & $2.5[1.5-4]$ & 0.139 & 0.001 & 0.022 \\
\hline
\end{tabular}

*, stands for <0.001. SAPS II, Simplified Acute Physiology Score II; SOFA, Sequential Organ Failure Assessment; I/O, input/output; SpO ${ }_{2}$, pulse oximetry; CVP, central venous pressure; CBC, complete blood count; CRP, C-reactive protein; ALT, alanine transaminase; AST, aspartate aminotransferase; BUN, blood urea nitrogen; $\mathrm{pH}$, blood urea nitrogen; $\mathrm{PO}_{2}$, partial pressure of oxygen; $\mathrm{PCO}_{2}$, partial pressure of carbon dioxide.

In the subgroup analysis of deep sedation patients, we found that patients with high MAP had a much higher mortality rate $(48.5 \%)$ compared with low and moderate blood pressure groups. According to clinical practice, as agitation/sedation medications may cause hypotension, doctors may use a relatively high dosage of vasoactive agents to prevent hypotension. Interestingly, our results showed that patients with higher blood pressure also had a worse prognosis. Thus, patients may not benefit from aimless blood pressure elevations. There may be several reasons for this. First, increasing arterial pressure does not necessarily improve microvascular perfusion. In 2015, the concept of "hemodynamic coherence" was examined by Ince (22). The goal of recovery is to improve systematic circulation and 
Table 3 Mortality rate of patients under different blood pressure patterns and sedation depth

\begin{tabular}{lccc} 
& Low MAP $(n=6,194)$ & Moderate MAP $(n=7,093)$ & High MAP $(n=1,498)$ \\
\hline Light sedation $(n=433)$ & 26.5 & 13.8 & 8.9 \\
Moderate sedation $(n=12,742)$ & 14.2 & 10.2 & 10.8 \\
Deep sedation $(n=1,610)$ & 33.7 & 35.7 & 48.5 \\
\hline
\end{tabular}

MAP, mean arterial pressure.

microcirculation simultaneously. In a study of 252 patients with severe sepsis for whom sublingual microcirculation was visualized, De Baker et al. found that circulation-perfusion uncoupling in microcirculation was strongly associated with an unsatisfactory prognosis (23). Second, different organs have different requirements for perfusion pressure under different hemodynamic conditions; fluctuations in blood pressure and their relationship with metabolism may be the most important parameters (24). Adrenergic vasopressors could cause a reduction in splanchnic circulation, which could increase the oxygen consumption of the intestinal tract and the whole body, which could, in turn, elevate lactic acid (25).

Further, adrenergic vasopressors are associated with reductions in cerebral tissue oxygenation (26), cardiac complications, including myocardial cell injury and atrial fibrillation (27), and muscle weakness in mechanically ventilated patients (28). Thus, the appropriate blood pressure should be determined based on tissue perfusion function and each patient's specific condition (29). Additionally, it may be that subjective evaluation methodologies of sedation (based on the Richmond-ASS and Riker Sedation-Agitation Scale) do not precisely reflect a patient's actual sedation status. An actual unsatisfactory sedation level could cause high MAP. In such circumstances, to rule out this possibility, objective evaluation method should be used to titrate the usage of agitation/sedation medications, such as bispectral index monitoring (30).

The limitations of this study need to be considered. First, vasoactive agents may have also contributed to changes in blood pressure. In the low blood pressure group, 1,810 patients used anti-hypertension agents, which is surprising, as anti-hypertension agents are seldom used in patients with low blood pressure. It may be that those patients had relatively high blood pressure initially, and their blood pressure only lowered after their physicians administered anti-hypertension agents. However, compared to moderate blood pressure, low blood pressure did not produce any benefits for patients. Additionally, in this study, $14.2 \%$ of patients used both vasopressors and hypotensive drugs in the first 24 hours in the ICU, which suggests that there is a modulation/titration in blood pressure in the first 24 hours. We do not have any information about the sequencing and dosage of vasopressors and hypotensive drugs for these patients. However, all adjustments of vasoactive agents could end up affecting blood pressure. Second, concerning the database, we were not aware of the real target MAP of each patient. Thus, in considering the reason why some patients had poor outcomes, we cannot determine whether the physicians achieved an optimal MAP for patients or whether the patients had a serious illness that prevented physicians from achieving the MAP target. Third, three group with low, moderate, and high MAP had different baseline characteristics, which could potentially affect outcome. Logistic regression can be used to make adjustments of confounding factors. However, given there are three sedation levels and three blood pressure levels, more than 25 parameters which may potentially affect outcome analysis and some of them are interactive (e.g., evaluation of SAPS II score including MAP. A low MAP may increase SAPS II. Evaluation of SOFA score including vasopressors, the usage of vasopressors may increase SOFA score), such measurements that reduce effect of confounding factors have its limitations too.

In terms of the machine learning analysis, the spectral clustering method does not assume the statistics of the clusters and is highly adaptable to different data distributions, including MAP data distributions. The main advantage of t-SNE is its ability to maintain a local structure, which suggests that similar points in the highdimensional data space remain similar when projected into a low-dimensional data space. A t-distribution has a longer tail, which helps the data points to be more evenly distributed in the 2-dimensional space.

\section{Conclusions}

We examined sedated critically ill patients on mechanical ventilation and found that patients with low MAP had 
a high possibility of a poor prognosis. High MAP in deep sedated patients should be cautiously considered to identify any underlying issues. To improve prognosis, a personalized MAP target should be set according to the severity of the illness and level of sedation. We should endeavor to use the positive hemodynamic effects of analgesic and sedative treatments to facilitate the treatment of the underlying disease in patients with critical illness to improve patient outcomes. Such medications may not only relieve pain and anxiety but also optimize tissue perfusion and oxygen supply and demand.

\section{Acknowledgments}

Funding: China Health Information and Health Care Big Data Association Severe Infection Analgesia and Sedation Big Data Special Fund (Grant No. Z-2019-1-001). Public Service Platform of Industrial Technology Foundation from the Ministry of Industry and Information Technology of China (Grant No. 2019-00893-1-2). China International Medical Foundation Analgesia and Sedation Special Fund (Grant No. Z-2017-24-2028-01). Excellence Program of Key Clinical Specialty of Beijing in 2020. Beijing Municipal Science and Technology Commission (Grant No. Z201100005520051).

\section{Footnote}

Reporting Checklist: The authors have completed the STROBE reporting checklist. Available at https://dx.doi. org/10.21037/atm-21-2806

Conflicts of Interest: All authors have completed the ICMJE uniform disclosure form (available at https://dx.doi. org/10.21037/atm-21-2806). The authors have no conflicts of interest to declare.

Ethical Statement: The authors are accountable for all aspects of the work in ensuring that questions related to the accuracy or integrity of any part of the work are appropriately investigated and resolved. This study is carried out according to the provisions of the Declaration of Helsinki (revised in 2013).

Open Access Statement: This is an Open Access article distributed in accordance with the Creative Commons Attribution-NonCommercial-NoDerivs 4.0 International License (CC BY-NC-ND 4.0), which permits the noncommercial replication and distribution of the article with the strict proviso that no changes or edits are made and the original work is properly cited (including links to both the formal publication through the relevant DOI and the license). See: https://creativecommons.org/licenses/by-nc-nd/4.0/.

\section{References}

1. Simons KS, Verweij E, Lemmens PMC, et al. Noise in the intensive care unit and its influence on sleep quality: a multicenter observational study in Dutch intensive care units. Crit Care 2018;22:250.

2. Darbyshire JL. Excessive noise in intensive care units. BMJ 2016;353:11956.

3. Mazeraud A, Polito A, Sivanandamoorthy S, et al. Association Between Anxiety and New Organ Failure, Independently of Critical Illness Severity and Respiratory Status: A Prospective Multicentric Cohort Study. Crit Care Med 2020;48:1471-9.

4. Barr J, Fraser GL, Puntillo K, et al. Clinical practice guidelines for the management of pain, agitation, and delirium in adult patients in the intensive care unit. Crit Care Med 2013;41:263-306.

5. Song Y, Gao S, Tan W, et al. Dexmedetomidine versus midazolam and propofol for sedation in critically ill patients: Mining the Medical Information Mart for Intensive Care data. Ann Transl Med 2019;7:197.

6. Kollef MH, Levy NT, Ahrens TS, et al. The use of continuous i.v. sedation is associated with prolongation of mechanical ventilation. Chest 1998;114:541-8.

7. Stephens RJ, Dettmer MR, Roberts BW, et al. Practice Patterns and Outcomes Associated With Early Sedation Depth in Mechanically Ventilated Patients: A Systematic Review and Meta-Analysis. Crit Care Med 2018;46:471-9.

8. Benken S, Madrzyk E, Chen D, et al. Hemodynamic Effects of Propofol and Dexmedetomidine in Septic Patients Without Shock. Ann Pharmacother 2020;54:533-40.

9. Noel-Morgan J, Muir WW. Anesthesia-Associated Relative Hypovolemia: Mechanisms, Monitoring, and Treatment Considerations. Front Vet Sci 2018;5:53.

10. Weerink MAS, Struys MMRF, Hannivoort LN, et al. Clinical Pharmacokinetics and Pharmacodynamics of Dexmedetomidine. Clin Pharmacokinet 2017;56:893-913.

11. Ebert TJ. Sympathetic and hemodynamic effects of moderate and deep sedation with propofol in humans. Anesthesiology 2005;103:20-4.

12. Meng $\mathrm{L}$, Yu W, Wang T, et al. Blood Pressure Targets in Perioperative Care. Hypertension 2018;72:806-817. 
13. Rhodes A, Evans LE, Alhazzani W, et al. Surviving Sepsis Campaign: International Guidelines for Management of Sepsis and Septic Shock: 2016. Intensive Care Med 2017;43:304-77.

14. Johnson AE, Pollard TJ, Shen L, et al. MIMIC-III, a freely accessible critical care database. Sci Data 2016;3:160035.

15. Akima H. A new method of interpolation and smooth curve fitting based on local procedures. JACM 1970;17:589-602.

16. Sessler CN, Gosnell MS, Grap MJ, et al. The Richmond Agitation-Sedation Scale: validity and reliability in adult intensive care unit patients. Am J Respir Crit Care Med 2002;166:1338-44.

17. Riker RR, Picard JT, Fraser GL. Prospective evaluation of the Sedation-Agitation Scale for adult critically ill patients. Crit Care Med 1999;27:1325-9.

18. Khan BA, Guzman O, Campbell NL, et al. Comparison and agreement between the Richmond Agitation-Sedation Scale and the Riker Sedation-Agitation Scale in evaluating patients' eligibility for delirium assessment in the ICU. Chest 2012;142:48-54.

19. Ng AY, Michael I, Weiss Y. On spectral clustering: Analysis and an algorithm. Advances in Neural Information Processing Systems 2002;2:849-56.

20. Rousseeuw PJ. Silhouettes: a graphical aid to the interpretation and validation of cluster analysis. Journal of Computational and Applied Mathematics 1987;20:53-65.

21. Maheshwari K, Nathanson BH, Munson SH, et al. The relationship between ICU hypotension and in-hospital mortality and morbidity in septic patients. Intensive Care Med 2018;44:857-67.

22. Ince $\mathrm{C}$. Hemodynamic coherence and the rationale for

Cite this article as: Liu S, Su L, Liu X, Zhang X, Chen Z, Liu C, Hong N, Li Y, Long Y. Recognizing blood pressure patterns in sedated critically ill patients on mechanical ventilation by spectral clustering. Ann Transl Med 2021;9(18):1404. doi: 10.21037/atm-21-2806 monitoring the microcirculation. Crit Care 2015;19 Suppl 3:S8.

23. De Backer D, Donadello K, Sakr Y, et al. Microcirculatory alterations in patients with severe sepsis: impact of time of assessment and relationship with outcome. Crit Care Med 2013;41:791-9.

24. Pinsky MR, Dubrawski A. Gleaning knowledge from data in the intensive care unit. Am J Respir Crit Care Med 2014;190:606-10.

25. Ensinger H, Weichel T, Lindner KH, et al. Effects of norepinephrine, epinephrine, and dopamine infusions on oxygen consumption in volunteers. Crit Care Med 1993;21:1502-8.

26. Yousef KM, Crago E, Chang Y, et al. Vasopressor Infusion After Subarachnoid Hemorrhage Does Not Increase Regional Cerebral Tissue Oxygenation. J Neurosci Nurs 2018;50:225-30.

27. Dünser MW, Hasibeder WR. Sympathetic overstimulation during critical illness: adverse effects of adrenergic stress. J Intensive Care Med 2009;24:293-316.

28. Wolfe KS, Patel BK, MacKenzie EL, et al. Impact of Vasoactive Medications on ICU-Acquired Weakness in Mechanically Ventilated Patients. Chest 2018;154:781-7.

29. Su LX, Liu DW. Personalized Critical Hemodynamic Therapy Concept for Shock Resuscitation. Chin Med J (Engl) 2018;131:1240-3.

30. Devlin JW, Skrobik Y, Gélinas C, et al. Clinical Practice Guidelines for the Prevention and Management of Pain, Agitation/Sedation, Delirium, Immobility, and Sleep Disruption in Adult Patients in the ICU. Crit Care Med 2018;46:e825-73. 